\title{
A realistic view of the cardiothoracic surgery specialty
}

Tomas A. SALERNO

RBCCV 44205-???

The following represents my views and experiences as a cardiovascular and thoracic surgeon. It is my hope that the reader will gain some insight into a specialty that has undergone major changes in the last decades.

When Gruntzig introduced angioplasty, I was just beginning my career in cardiovascular and thoracic surgery. I attended one of his lectures - a presentation that captured the imagination of cardiologists and surgeons differently. Surgeons were skeptical about this new procedure. They were used to seeing small arteries in the operating room and questioned how one would be able to introduce a small catheter into the femoral artery, negotiate it via the left main coronary artery into a distal vessel and dilate it.

Cardiologists, who were not used to seeing arteries in the operating room, but rather had viewed them in two dimensions with dye introduced via a catheter, saw this as an incredible opportunity to treat patients with ischemic heart diseases. What followed was to become one of the most successful treatment modalities for ischemic heart disease. Unfortunately, surgeons did not actively participate in this important development and played an emergency "back up" role for this procedure.

Initially there was a learning curve for angioplasty, with large number of patients undergoing emergency coronary artery bypass surgery due acute occlusion of the coronary artery. At the time, there was uncertainty in the minds of surgeons as to the future of this new procedure. Many believed that there was going to be an epidemic of either acute failures or short/long term stenoses, overburdening the already crowded operating rooms. It was suggest that there may be the need to increase the number of operating rooms in some hospitals, as we braced for an epidemic of patients in need of urgent myocardial revascularization. Until that time, coronary artery surgery had been the only method of myocardial revascularization. Moreover, angioplasty was quickly followed by better ways to treat stenoses with the development of coronary stents. Who would ever think that one could place a small coil into one of these small arteries and achieve satisfactory clinical results? Cardiologist now had a procedure that was simple, effective and that could restore health almost instantaneously without the need of surgical intervention. This is all history now. I recently attended a cardiology meeting in which a senior cardiologist made the statement that there were practically no patients that needed surgical revascularization. He simply wanted the surgeon to standby just in case the patient "crashed".

With the passage of time, surgeons have abandoned some aspects of our specialty. I remember being one of the busiest surgeons doing rigid and then flexible bronchospies, a 
practice which was subsequently taken over by the pulmonologists. Now, only at time of surgery do we perform bronchoscopy. The diagnostic and investigative components of patient care have been delegated to other physicians. Similarly, pacemaker and ICDs became the realm of the "interventionalists" and few surgeons currently perform these procedures.

As incisions got smaller, surgeons relegated invasive procedures to other specialities. There was a time when ablation therapy for ventricular arrhythmias was in the domain of the cardiac surgeons. The surgeons actually helped developed the technology of catheter ablation, which ultimately eliminated their involvement. Why were the surgeons not involved in catheter ablation? Again, this procedure was relegated to the "interventionalists". I guess the catheterization laboratory was no place for surgeons. Vascular surgery became a separate specialty, with its own certificate and requirements. Residency training programs now became "cardiothoracic", and vascular surgery was no longer part of it. And finally, even cardiac surgery and thoracic surgery have begun to separate. Few cardiothoracic surgeons operate upon the heart, esophagus and lungs. Some of us do just non-cardiac thoracic; the remainder of us are simply coronary artery surgeons, standing-by for the cardiologists who treat the majority of these patients. Valvular procedures are few and it is not uncommon for some surgeons to perform ten or less such procedures each year. Furthermore, cardiologists begun to develop new methods to treat valvular diseases in the catheterization laboratory, such as percutaneous mitral valvuloplasty.

In the end, cardiac surgery has really become coronary artery surgery. Perhaps even more sad is the fact that we have practically lost our patients. Patients are fully worked up by the cardiologists and are referred to us for a specific operation, dictated by the cardiologists. After surgery, the postoperative care is frequently delegated to intensivists or cardiologists, and thereafter, follow-up is by the cardiologists. If surgeons were to develop a new procedure to surgically treat ischemic heart disease, they might not be able to recruit patients, since they have long lost our primary referral.

Where is the future? I think about the controversy surrounding off-pump coronary surgery. When the LIMAto-LAD via left thoracotomy became available, the biggest advocates of this procedure were the cardiologists. Surgeons were hesitant in accepting the possibility of performing this operation without the heart-lung machine and via smaller incisions.

The same applies today for off-pump coronary artery revascularization, which is only performed in about $20 \%$ of centers across the United States. The most critical of offpump coronary surgery are the surgeons, who continue to be reluctant to adopt this new technology. Why change when we have an excellent operation? If cardiologists had behaved in the same way, advances in angioplasty/stenting would have come to a halt. The cardiologists had the vision, however, and seized the opportunity. They seem to have welcome changes in technology that has allowed them to develop new procedures and to treat patients effectively. It may be too late to regain some of ground that has been lost. We will never be able to regain control of angioplasty/ stents, ICDs, pacemakers, ablation therapy and others. The specialty is predominantly cardiac and, in that specialty, coronary artery surgery is literally the sole operation that some surgeons perform.

We must develop a vision for the future and at least consider being involved in new modalities of therapy for the treatment of patients with heart diseases. We must regain control of our patients and at least make an attempt at developing a referral pattern whereby patients are primarily referred to the surgeon for an evaluation. If we look at some of the future developments, such as heart failure treatment, again we are facing the same issues as in coronary artery surgery. Most of these patients are treated by physicians; surgeons rarely see the patients as the primary doctor.

There is a great potential for our future, if we learn lessons from the past. There has been an explosion of new developments in cardiac surgery such as robotics, minimally invasive valve and coronary artery surgery, a variety of surgical procedures for the treatment of dilated cardiomyopathy, totally implantable assist devices and artificial heart, gene therapy for ischemic heart disease, myocyte replacement of the damaged heart, stents for a variety of conditions such as stenoses of different arteries and aneurysms of the aorta, transmyocardial revascularization, and others.

It is with regret that I already see that some of these procedures are already being taken over by other specialists such as radiologists, cardiologists, neurosurgeons, while surgeons continue to be interested primarily in the operating room. I know of a center where an endovascular division was created, headed by neurosurgeons, who perform a variety of stent procedures in vessels such as the carotids, subclavian, thoracic and distal aorta, and peripheral arteries! Where were the cardiac and vascular surgeons?

For those of us coming to a late phase in our professional lives this probably will not matter. For those initiating a career in cardiac surgery, they will have a lot to lose unless they get involved in these new developments. Being a surgeon is not necessary spending all one's time in the operating room. Furthermore, it is time that surgeons should not feel that it is beneath them to perform lesser procedures other than heart surgery.

In my opinion, it is time that we face reality and take steps to correct some of these omissions in our clinical practice, since most cardiologists consider us mere technicians. 